\title{
Spatial Analysis of Urbanization in Major Asian and African Cities
}

\author{
Manjula Ranagalage ${ }^{\mathrm{a}, \mathrm{b} *}$, Yuji Murayama ${ }^{\mathrm{c}}$ \\ ${ }^{a}$ Graduate School of Life and Environmental Sciences, University of Tsukuba, 1-1-1, Tennodai, Tsukuba, Ibaraki 305-8572, Japan; \\ manjularanagalage@gmail.com \\ ${ }^{b}$ Department of Environmental Management, Faculty of Social Sciences and Humanities, Rajarata University of Sri Lanka, \\ Mihintale 50300, Sri Lanka \\ ${ }^{c}$ Faculty of Life and Environmental Sciences, University of Tsukuba, 1-1-1, Tennodai, Tsukuba, Ibaraki 305-8572, Japan; \\ mura@geoenv.tsukuba.ac.jp \\ * Manjula Ranagalage
}

Keywords: spatial pattern, urbanization, Asia, Africa, web-based systems

Abstract:

The world statistics shows that $54 \%$ of the world population had been accumulated in urbanized areas by 2014 . It is estimated that the global urban population will be increasing up to $66 \%$ of the total by 2050 . According to the United Nations (UN) projection, the urbanization will be faster in Asian and African countries than the other continents. It is obvious this rapid increase will bring about serious socio-economic and environmental problems in the near future. Thus the geographical thinking of urbanization is becoming a vital topic to introduce proper urban planning for the future sustainability.

Many geographers have focused on the urbanization pattern and process of the developing countries during the last two decades. In this connection, the scarcity of the spatial data has been an obstacle to study quantitatively urbanization especially in Asian and African cities. Based on this background, since the 2000s, the Division of Spatial Information Sciences, University of Tsukuba, has conducted the research to establish the city-based spatial information platform to overcome this obstacle, i.e., the lack of the data. The main objective of this project is to capture the urbanization pattern and process over the time by using Remote Sensing (RS) and Geographical Information Systems (GIS) techniques. The data on land use and land cover, land surface temperature, energy consumption, and population density are now available with the same spatial resolution in terms of selected cities.

Our goal is to open the web-based GIS system to provide the geospatial database of Asian and African cities and scientifically discuss the urban sustainability by using scenario-based simulation. The projected future scenario will be useful for the urban policy by the "back-casting method." 